\title{
2||-||||||||||||||||||||||||||||||||||||||||||||||||||||||||||||||||||.
}

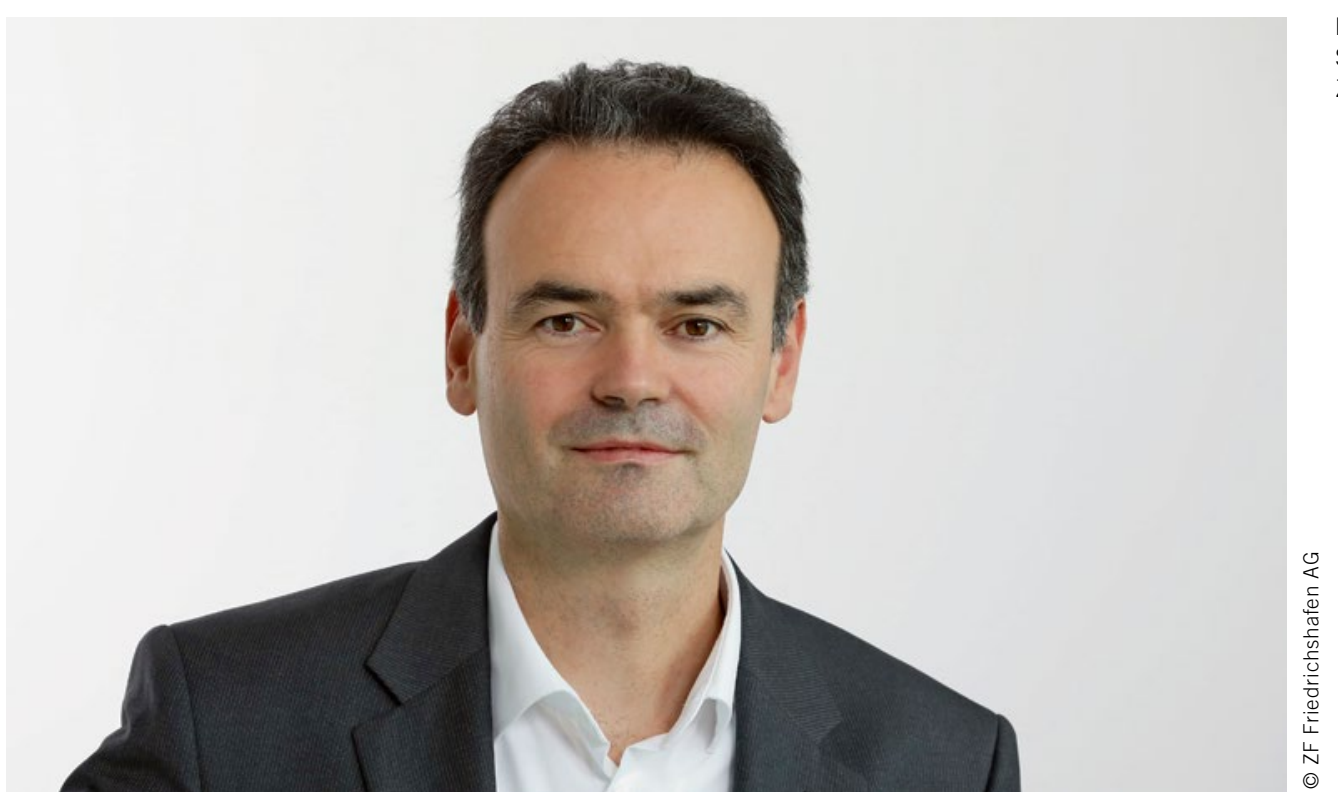

Dr. Otmar Scharrer

Senior Vice President R\&D

ZF Friedrichshafen AG

\section{Elektromobilität geht in die entscheidende Phase}

Elektromobilität hat heute verschiedene Ausprägungen. Dem PHEV-Konzept kommt eine hohe Bedeutung für die nahe Zukunft zu, denn nur diese Antriebsform ist aktuell in der Lage, die Kundenanforderungen in idealer Weise in einem einzigen Auto zu verbinden: elektrisches und damit lokal emissionsfreies Fahren in der Stadt bei gleichzeitig ausreichender Reichweite auf der Langstrecke. Voraussetzung dafür ist ein hinreichend großer Batteriespeicher, der in den allermeisten Hybridfahrzeugen der neuen Generation vorhanden sein wird. Dieses Konzept haben wir bei ZF unter dem Namen EVplus mit einer gesicherten elektrischen Reichweite von $80 \mathrm{~km}$ bereits vor einigen Jahren als voll funktionsfähigen Erprobungsträger vorgestellt.

Bei den Antrieben für BEV ist eine große Anzahl von Varianten im Feld, und aktuell sind viele weitere in der Entwicklung. Auffällig ist hier die große technische Vielfalt. Das trifft schon für den Elektromotor zu, bei dem über verschiedene Motorbauformen und Wicklungsarten der Maschine der ganze Bogen an Möglichkeiten aufgespannt wird. Das gilt auch für die Leistungselektronik, bei der heute zahlreiche unterschiedliche Leistungsmodule in unterschiedlichen Spannungslagen verbaut werden. Deren Anzahl dürfte sich mit der Siliziumkarbid-Technik noch weiter erhöhen. Zuletzt gibt es beim Achsenlayout sowohl koaxiale als auch achsparallele Systeme in unterschiedlichen Einbauformen als Ein- oder Mehrgänger. Für die Hersteller wie die Zulieferer stellt sich daher die Frage nach einer sinnvollen
Standardisierung. Die OEMs werden im Einzelfall sicherlich eine Make-or-Buy-Analyse durchführen und durch die jeweilige Entscheidung ihren technologischen Footprint festlegen. Für die Zulieferer ist es hingegen von großer Bedeutung, zum richtigen Zeitpunkt einen Technologiesprung zu vollführen, bei dem möglichst viele vorhandene Innovationen so in ein neues, modulares Konzept eingebettet werden, in dem auch individuelle Merkmale für spezifische Kundenwünsche realisiert werden können.

Die Automobilindustrie entwickelt in den meisten Teilen der Welt gerade die letzte neue Antriebsgeneration mit einer Verbrennungskraftmaschine. Auf die Elektromobilität wird dadurch ein starker Impuls ausgeübt. Nicht zuletzt durch die Corona-Pandemie kam es zu einer weiteren Beschleunigung dieser Aktivitäten. Die Elektromobilität geht daher in die entscheidende Phase, in der sie die verbrennungsmotorische Mobilität ablösen wird. Der Schlüssel zum Erfolg wird neben den Kosten die erzielbare Reichweite bei gegebener Batteriegröße sein. Neben den erwähnten Hardwarelösungen kommen in der nächsten Generation auch erfolgskritische Softwarekomponenten auf den Markt, die durch prädiktive Abschätzung von Verkehrssituationen großen Anteil an der Effizienz haben werden. Zusätzliche Funktionen für den Kunden wie Vehicle-to-Grid runden das Angebot dieser nächsten BEV-Generation ab. Der Systemgedanke wird für diese Phase wichtiger denn je, um sich mit der erforderlichen Geschwindigkeit an sich ändernde Randbedingungen anpassen zu können. 